\title{
NOTE ON THE HURWITZ ZETA-FUNCTION ${ }^{1}$
}

\author{
N. J. FINE
}

It is well known that Riemann ${ }^{2}$ gave two proofs of the functional equation for $\zeta(s)$, the first depending on a contour integration, the second on the transformation equation for $\vartheta_{3}(0 \mid \tau)$. Hurwitz $z^{3}$ introduced his generalized zeta-function, defined for $R(s)>1$ and $0<a<1$ by $^{4}$

$$
\zeta(s, a)=\sum_{n=0}^{\infty}(n+a)^{-s},
$$

showed that it can be continued to the entire $s$-plane with the exception of a simple pole at $s=1$, and proved that for $R(s)>1$,

$$
\begin{aligned}
\zeta(1-s, a)= & \frac{2 \Gamma(s)}{(2 \pi)^{s}}\left\{\cos \frac{\pi s}{2} \sum_{n=1}^{\infty} \frac{\cos 2 n \pi a}{n^{s}}\right. \\
& \left.+\sin \frac{\pi s}{2} \sum_{n=1}^{\infty} \frac{\sin 2 n \pi a}{n^{s}}\right\} .
\end{aligned}
$$

His method of proof depends on a contour integral and parallels Riemann's first proof. It appears to have been overlooked that the second method of Riemann can be generalized to obtain the same results. ${ }^{5}$ The purpose of this paper is to supply such a proof.

For $0<a<1$ and $x>0$, define

$$
\begin{aligned}
f(a, x) & =\vartheta_{3}(\pi a, i x) \\
& =1+2 \sum_{n=1}^{\infty} e^{-\pi n^{2} x} \cos 2 \pi n a .
\end{aligned}
$$

By the transformation equation for the $\vartheta$-function, ${ }^{6}$

Received by the editors June 10, 1950.

${ }_{1}^{1}$ This work is an offshoot of investigations carried out under the auspices of the Office of Naval Research, Contract N9-ONR90,000.

${ }^{2}$ B. Riemann, Ueber die Anzahl der Primzahlen unter einer gegebenen Grösse, Monatsberichte der Preussischen Akademie der Wissenschaften $(1859,1860)$ pp. 671680.

${ }^{3}$ A. Hurwitz, Zeitschrift für Mathematik und Physik vol. 27 (1882) p. 95.

- Throughout this paper, $x^{s}=\exp (s \log x)$, the logarithm being real for $x>0$.

${ }^{5}$ R. Lipschitz (J. Reine Angew. Math. vol. 105, pp. 127-159) has used the thetafunction transformation device to derive a functional equation for a general type of zeta-function, but his results do not appear to include ours.

- Whittaker and Watson, Modern analysis, p. 475. 


$$
f\left(a, x^{-1}\right)=x^{1 / 2} e^{-\pi a^{2} x} f(\text { iax }, x)=x^{1 / 2} \sum_{n=-\infty}^{+\infty} e^{-\pi x(n+a)^{2}} .
$$

From (3) and (4) it is easy to see that $f(a, x)$ tends to the values 1 and 0 exponentially as $x$ tends to $\infty$ and 0 , respectively. Hence the two functions

$$
\begin{aligned}
& F(a, s)=\int_{0}^{1} f(a, x) x^{s / 2-1} d x \\
& G(a, s)=\int_{1}^{\infty}(f(a, x)-1) x^{s / 2-1} d x
\end{aligned}
$$

are entire in $s$. We define

$$
H(a, s)=F(a, s)+G(a, s)-2 / s .
$$

For a later purpose, we observe that

$$
\frac{\partial}{\partial a} H(a, s)=\int_{0}^{\infty} \frac{\partial}{\partial a} f(a, x) x^{s / 2-1} d x
$$

is also an entire function of $s$.

Now for $R(s)>1$,

$$
\begin{aligned}
H(a, s) & =\int_{0}^{\infty}(f(a, x)-1) x^{s / 2-1} d x \\
& =2 \sum_{n=1}^{\infty} \cos 2 \pi n a \int_{0}^{\infty} e^{-\pi n^{2} x} x^{s / 2-1} d x, \\
H(a, s) & =2 \pi^{-s / 2} \Gamma\left(\frac{s}{2}\right) \sum_{n=1}^{\infty} \frac{\cos 2 \pi n a}{n^{s}} \quad(R(s)>1) .
\end{aligned}
$$

For $R(s)<0$,

$$
G(a, s)=\frac{2}{s}+\int_{1}^{\infty} f(a, x) x^{s / 2-1} d x
$$

so that

$$
\begin{aligned}
H(a, s) & =\int_{0}^{\infty} f(a, x) x^{s / 2-1} d x \\
& =\int_{0}^{\infty} f\left(a, x^{-1}\right) x^{-s / 2-1} d x .
\end{aligned}
$$

By (4), therefore, 


$$
H(a, s)=\sum_{n=-\infty}^{+\infty} \int_{0}^{\infty} e^{-\pi x(n+a)^{2}} x^{(1-s) / 2-1} d x
$$

(10) $\quad H(a, s)=\pi^{-(1-s) / 2} \Gamma\left(\frac{1-s}{2}\right) \sum_{n=-\infty}^{+\infty}|n+a|^{-(1-s)} \quad(R(s)<0)$.

With $s$ replaced by $1-s,(10)$ becomes

$$
\begin{aligned}
H(a, 1-s) & =\pi^{-s / 2} \Gamma\left(\frac{s}{2}\right)\left\{\sum_{n=0}^{\infty}(n+a)^{-s}+\sum_{n=0}^{\infty}(n+1-a)^{-s}\right\} \\
& =\pi^{-s / 2} \Gamma\left(\frac{s}{2}\right)\{\zeta(s, a)+\zeta(s, 1-a)\} \quad(R(s)>1) .
\end{aligned}
$$

Now it is easy to see, from (1), that

$$
\frac{\partial}{\partial a} \zeta(s, a)=-s \zeta(s+1, a)
$$

Hence, differentiating (11) and replacing $s$ by $s-1$, we have

$$
\begin{array}{rlr}
\frac{\partial}{\partial a} H(a, 2-s)=- & (s-1) \pi^{-(8-1) / 2} \Gamma\left(\frac{s-1}{2}\right)\{\zeta(s, a) & \\
& -\zeta(s, 1-a)\} & (R(s)>2) .
\end{array}
$$

Combining (11) and (12) yields

$$
\begin{aligned}
2 \zeta(s, a)= & \frac{\pi^{s / 2}}{\Gamma(s / 2)} H(a, 1-s) \\
& -\frac{\pi^{(s-1) / 2}}{(s-1) \Gamma((s-1) / 2)} \frac{\partial}{\partial a} H(a, 2-s) \quad(R(s)>2) .
\end{aligned}
$$

Equation (13) provides the analytic continuation of $\zeta(s, a)$, and shows that $\zeta(s, a)-(s-1)^{-1}$ is an entire function of $s$. Replace $s$ by $1-s$ in (13):

$$
\begin{aligned}
2 \zeta(1-s, a)= & \frac{\pi^{(1-s) / 2}}{\Gamma((1-s) / 2)} H(a, s) \\
& +\frac{\pi^{-s / 2}}{s \Gamma(-s / 2)} \frac{\partial}{\partial a} H(a, 1+s) .
\end{aligned}
$$

For $R(s)>1$, we may use (9) to get

$$
\frac{\partial}{\partial a} H(a, 1+s)=-4 \pi^{(1-s) / 2} \Gamma\left(\frac{1+s}{2}\right) \sum_{n=1}^{\infty} \frac{\sin 2 \pi n a}{n^{s}} .
$$


Substituting from (9) and (15) into (14), and simplifying by well known formulas for the $\Gamma$-function, we obtain the desired relation (2).

The proof just presented does not cover the classical Riemann zeta-function, defined, for $R(s)>1$, by

$$
\zeta(s)=\sum_{n=1}^{\infty} n^{-s} .
$$

But it is easy to see that, for $R(s)>1$,

$$
\begin{aligned}
\zeta\left(s, \frac{1}{2}\right) & =\sum_{n=0}^{\infty}\left(\frac{2 n+1}{2}\right)^{-s}=2^{s} \sum_{n \text { odd }} n^{-s} \\
& =2^{s}\left\{\sum_{n=1}^{\infty} n^{-s}-\sum_{n=1}^{\infty}(2 n)^{-s}\right\} \\
& =\left(2^{s}-1\right) \zeta(s) .
\end{aligned}
$$

This provides the continuation of $\zeta(s)$ and shows that it has a simple pole at $s=1$, with possible simple poles at $s=2 n \pi i / \log 2(n=0$, $\pm 1, \pm 2, \cdots)$. Now if we set $a=1 / 2$ in equation (2), we obtain, for $R(s)>1$,

$$
\begin{aligned}
\zeta\left(1-s, \frac{1}{2}\right) \frac{(2 \pi)^{s}}{2 \Gamma(s) \cos (\pi s / 2)} & =\sum_{n=1}^{\infty}(-1)^{n} n^{-s}=\sum_{n \text { even }} n^{-s}-\sum_{n \text { odd }} n^{-s} \\
& =2 \sum_{n=1}^{\infty}(2 n)^{-s}-\sum_{n=1}^{\infty} n^{-s} \\
& =\left(2^{1-s}-1\right) \zeta(s) .
\end{aligned}
$$

Using (16) with $s$ replaced by $1-s$, we get the required functional equation for $\zeta(s)$,

$$
\zeta(1-s)=\frac{2 \Gamma(s) \cos (\pi s / 2)}{(2 \pi)^{s}} \zeta(s) .
$$

Since the right side of (17) is regular at $s=1-2 n \pi i / \log 2$, the only singularity of $\zeta(s)$ is the simple pole at $s=1$.

University of Pennsylvania 